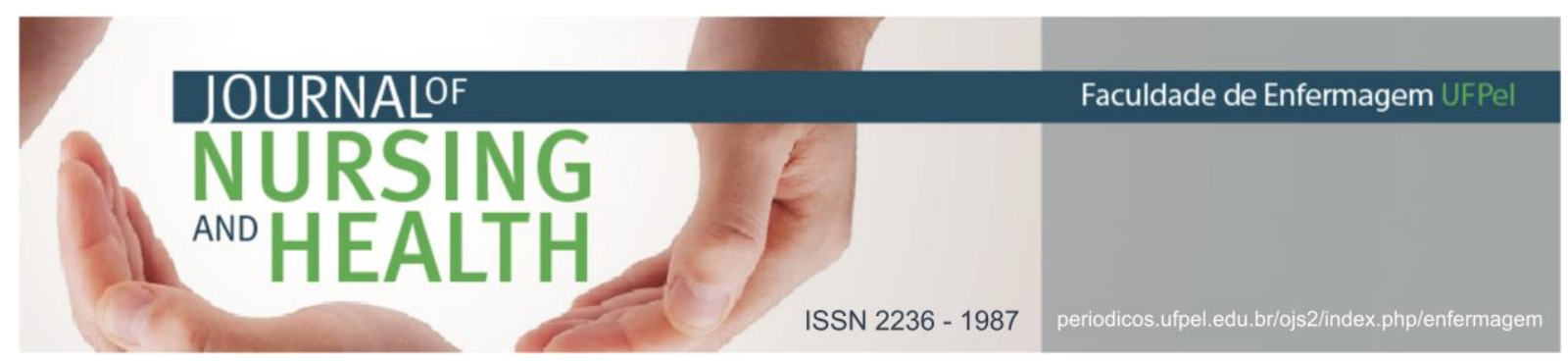

REVISÃO INTEGRATIVA

\title{
Intervenções não farmacológicas ofertadas ao usuário com transtorno mental comum na atenção primária à saúde
}

\author{
Non-pharmacological interventions offered to the user with a common mental \\ disorder in primary health care
}

\section{Intervenciones no farmacológicas ofrecidas al usuario con trastorno mental común en la atención primaria}

Chaves, Suellen Cristina da Silva ${ }^{1}$; Nobrega, Maria do Perpétuo Socorro de Sousa ${ }^{2}$; Silva, Taires dos Santos ${ }^{3}$

Como citar este artigo: Chaves SCS, Nobrega MPSS, Silva TS. Intervenções não farmacológicas ofertadas ao usuário com transtorno mental comum na atenção primária à saúde. J. nurs. health. 2019;9(3):e199302

\section{RESUMO}

Objetivo: analisar na literatura as intervenções não farmacológicas ofertadas aos indivíduos com Transtorno Mental Comum atendidos na Atenção Primária à Saúde. Métodos: trata-se de uma revisão integrativa, realizada no mês de maio e junho de 2018, com análise de publicações indexadas em bases de dados, nas línguas portuguesa, inglesa e espanhola, disponíveis gratuitamente, sem limitação da data de publicação. Resultados: foram selecionados cinco estudos que correspondiam aos critérios de inclusão e exclusão, sendo divididos em quatro temas principais: Terapia Cognitiva Comportamental; Terapia de resolução de problemas; Socioterapia e Cuidados Colaborativos. Considerações finais: os resultados ressaltam a escassez de estudos voltados para essa temática na literatura mundial e a necessidade do uso de terapias não farmacológicas como fator de consolidação da qualidade dos serviços prestados na Atenção Primária à Saúde.

Descritores: Transtornos mentais; Atenção primária à saúde; Terapias complementares.

\section{ABSTRACT}

Objective: to analyze in the literature the non-pharmacological interventions offered to individuals with Common Mental Disorder treated in Primary Health Care. Methods: this is an integrative review, carried out in May and June of 2018, with analysis of publications indexed in databases, in the Portuguese, English and Spanish languages, available free of charge, without limitation of publication date. Results: five studies were selected that corresponded to the inclusion and exclusion criteria, being divided into four main themes: Behavioral cognitive therapy; Problemsolving therapy; Socio-Therapy and Collaborative care. Final Considerations: the results highlight the scarcity of studies addressing this theme in the world literature and the need for the use of nonpharmacological therapies as a factor to consolidate the quality of services provided in Primary Health Care.

Descriptors: Mental disorders; Primary health care; Complementary Therapies.

1 Enfermeira. Universidade de São Paulo (USP). E-mail: smchaves@usp.br http: / / orcid.org/0000-0003-3234-9752 2 Enfermeira. Doutora em Enfermagem. Universidade de São Paulo (USP). E-mail: perpetua.nobrega@usp.br. http: / / orcid.org/0000-0002-5993-1446

3 Discente do curso de Enfermagem. Universidade de São Paulo (USP). E-mail: santostaires@gmail.com http://orcid.org/0000-0001-7840-4644 


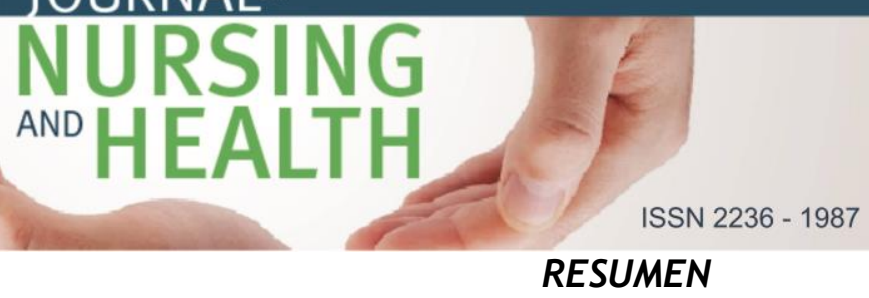

\section{RESUMEN}

Objetivo: analizar en la literatura las intervenciones no farmacológicas ofrecidas a los individuos con Trastorno Mental Común atendidos en la Atención Primaria a la Salud. Métodos: se trata de una revisión integrativa, realizada en el mes de mayo y junio de 2018, con análisis de publicaciones indexadas en bases de datos, en portugués, inglés y español, disponible de forma gratuita, pero sin limitarse a la fecha de publicación. Resultados: fueron seleccionados cinco estudios que correspondían a los criterios de inclusión y exclusión, siendo divididos en cuatro temas principales: Terapia cognitiva comportamental; Terapia de resolución de problemas; Socioterapia y Cuidados colaborativos. Consideraciones finales: los resultados resaltan la escasez de estudios orientados hacia esa temática en la literatura mundial y la necesidad del uso de terapias no farmacológicas como factor de consolidación de la calidad de los servicios prestados en la Atención Primaria a la Salud.

Descriptores: Trastornos mentales; Atención primaria de salud; Terapias complementarias.

\section{INTRODUÇÃO}

Os cuidados em Saúde Mental (SM) perpassam todos os níveis de atenção à saúde e devem ser fortalecidos de modo universal. No Brasil, a Política Nacional de Saúde Mental (PNSM), ancorada pela Lei $\mathrm{n}^{\circ}$ $10.216 / 2001$, vem se estruturando com a perspectiva de materialização da Rede de Atenção Psicossocial (RAPS) em um modelo de atenção de base comunitária. A Atenção Primária à Saúde (APS), como um dos componentes da RAPS, representa um lócus fértil para garantir o acesso aqueles que necessitam de cuidados de SM. ${ }^{1}$

A APS recebe demandas de pessoas com transtornos mentais de diversas gravidades, ${ }^{2}$ dentre essas, destacam-se os Transtornos Mentais Comuns (TMC) caracterizados por queixas somáticas inespecíficas, sem característica psicótica, que não preenchem critérios para os transtornos depressivos, ansiosos ou somatoformes de acordo com a Classificação Internacional de Doenças (10 ${ }^{\mathrm{a}}$ versão) e Diagnóstico e Estatística dos Transtornos Mentais (DSM IV).
Em termos epidemiológicos, a prevalência de TMC é bastante elevada. Globalmente, as taxas variam de $24,6 \%$ a $45,3 \%$ e no Brasil oscila de $28,7 \%$ a $50 \%$. Com estimativas para 2030 entre as perturbações mais incapacitantes do ser humano acometendo ambos os sexos, com maior propensão na população feminina, idosos e indivíduos com baixo nível socioeconômico e escolar, ${ }^{3-}$ ${ }^{6}$ exigindo oferta de cuidados médicopsicológico, além de outras ações terapêuticas.

Porém, observa-se que há dificuldade de compreensão da expressão de sofrimento em pessoas com TMC, isto ocorre devido a inaptidão das equipes e serviços no reconhecimento do sofrimento psíquico, ${ }^{7}$ pois a formação dos profissionais de saúde muitas vezes não atende as necessidades de saúde mental fora do campo da especialidade. Os desafios nesse campo são a superação da supremacia medicalizante para o sofrimento psíquico e a sedimentação de ações por meio da alteridade e sensibilização com o outro, desfavorecendo a 


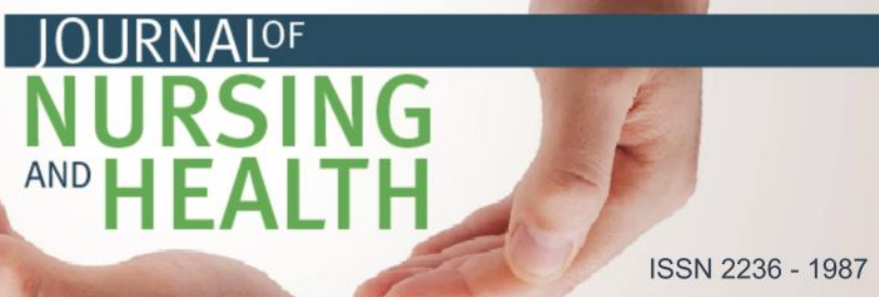

\section{MATERIAIS E MÉTODOS}

identificação e a prestação de cuidados precocemente na APS em relação aos TMC. $^{2}$

Culturalmente, a medicalização acompanha o homem desde o nascer até sua finitude. A supervalorização da farmacologia assume a excelência no alívio do sofrimento físico e psíquico e apresenta-se como quase insubstituível frente a outras medidas terapêuticas. Ainda que, reconhecendo as potencialidades da farmacologia, entende-se que as Intervenções Não Farmacológicas (INF) têm o poder de promover saúde e conforto à vida de indivíduos. ${ }^{6-8}$

As INF dizem respeito a um conjunto de estratégias que visam maximizar o funcionamento cognitivo e o bem-estar da pessoa, bem como ajudá-la no processo de adaptação à doença. As atividades desenvolvidas têm como fim a estimulação das capacidades individuais, preservando, pelo maior período possível sua autonomia, conforto e dignidade. ${ }^{8}$

Partindo do princípio que há fragilidade no reconhecimento do TMC e pouco investimento em medidas de suporte para pessoas com essa condição no campo da APS, e tendo como pressuposto que a medicalização do sofrimento psíquico permanece como a mais empregada neste cenário, este estudo contribui para uma outra perspectiva de cuidado e subsidia a melhora na qualidade da assistência a essa população. Assim, tem-se como objetivo analisar na literatura as intervenções não farmacológicas ofertadas aos indivíduos com TMC atendidos na Atenção Primária à Saúde.
Trata-se de uma revisão integrativa da literatura, espécie de revisão de natureza complexa, com métodos normatizados e sistemáticos que garantem o rigor indispensável e a legitimidade às evidências estabelecidas na pesquisa científica. ${ }^{9-}$ 11

Este método proporciona a avaliação crítica e síntese das evidências disponíveis sobre o tema investigado com base em pesquisas já publicadas. Identificam-se tendências da produção e a incorporação da aplicabilidade dos resultados de estudos significativos na prática e apontam-se lacunas que direcionam para o desenvolvimento de futuras pesquisas, sempre pautadas na análise ampla e sistemática de estudos científicos. ${ }^{11}$

As etapas da revisão foram: construção da questão norteadora, definição de critérios para levantamento na literatura, definição dos dados a serem extraídos, avaliação para inclusão, interpretação e apresentação dos resultados.

Para a formulação da questão norteadora e refutação dos elementos metodológicos fundamentais, utilizouse a pergunta PICo. Tal acrômio significa P - população, I - Intervenção, Co - Contexto/desfecho. ${ }^{10-11} \mathrm{Na}$ presente revisão, a estratégia mnemônica PICo foi selecionada da seguinte maneira: $P$ - população com transtorno mental comum, I intervenções terapêuticas não farmacológicas e Co - Atenção Primária à Saúde. Logo, a questão norteadora foi: Quais são as intervenções não 


\section{JOURNALOF \\ NURSING \\ ${ }^{\text {ANO }} \mathrm{HEALTH}$}

ISSN 2236 - 1987

Terapia Farmacológica (Drug Therapy). A fim de otimizar a busca de material, utilizou-se como fórmula estratégica as palavras-chaves: ("Primary Health Care" AND "Common Mental Disorders") NOT ("Drug Therapy").

Para a análise dos artigos, houve a elaboração de um instrumento pelas autoras e para análise das publicações, adotou-se o critério de independência com duas revisoras em todas as etapas.

\section{RESULTADOS}

$\mathrm{Na}$ busca inicial foram encontradas 848 publicações. Após análise rigorosa do título e resumo foram selecionados 51 artigos. A partir desta seleção procedeu-se à leitura destes na íntegra. A amostra final resultou em cinco artigos que tratam de intervenções terapêuticas não farmacológicas conduzidas em pessoas com TMC. 0 percurso metodológico dos procedimentos de busca nas bases de dados, seleção e inclusão dos artigos está representado no Quadro 1.

Observa-se, no Quadro 1, um número significativo de estudos que abordam o TMC, contudo, grande parte aborda este fenômeno sob a perspectiva das intervenções farmacológicas, evidenciando assim escassez de estudos que lidem com a questão sob um novo ponto de vista.

A partir dos estudos primários incluídos na revisão, elaborou-se um quadro contendo as seguintes características: ano/base de dados, autor(es), título, objetivo/amostra e conclusão, Quadro 2.
- Atenção Primária à Saúde (Primary Health Care), Transtorno Mental Comum (Common Mental Disorders) e 


\section{NURSING \\ AND HEALTH}

ISSN 2236 - 1987

Quadro 1: Distribuição dos artigos encontrados, excluídos e selecionados nas bases eletrônicas de dados. São Paulo, SP. Brasil, 2018.

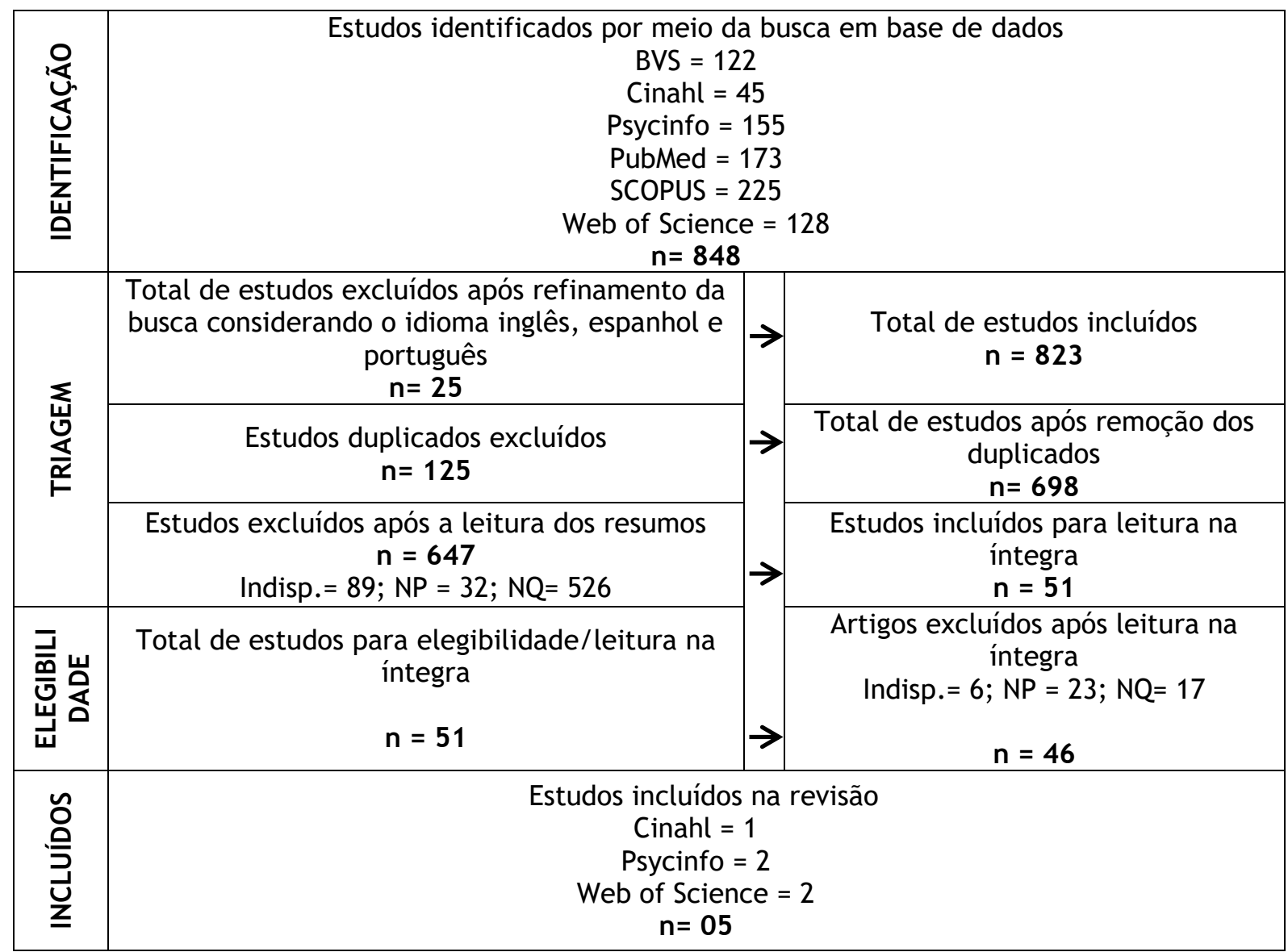

Legenda: $\mathrm{NP}=$ não é pesquisa; $\mathrm{NQ}=$ não responde às questões; Indisp. = Artigo indisponível eletronicamente na íntegra.

Os cinco artigos incluídos nesta revisão se distribuem nas seguintes bases de dados: Web of Science* (2) e

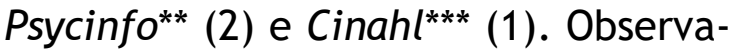
se que as publicações foram maiores no ano de 2011, correspondendo a um total de 60\% ( $n=2)$ e entre 2002, 2010 e 2015 com 40\% das publicações sendo uma publicação em cada ano. Todos publicados em língua inglesa, com metodologia quantitativa. Em relação à origem, foi significativo o número de produções provenientes de países europeus $(60 \%)$ e africanos (40\%).

Muitos dos artigos excluídos nessa revisão lidavam com o fenômeno do sofrimento psíquico sob a lógica medicamentosa unicamente, ou até mesmo, em associação com intervenções não farmacológicas, porém, demonstrando dificuldade quanto ao distanciamento da vertente biomédica. Já os estudos que compuseram esta revisão são de extrema relevância, uma vez que oferecem a possibilidade de sustentar a pessoa em sofrimento psíquico por meio de uma conduta não medicamentosa, e ainda abre canal para que essas abordagens possam ser reproduzidas na APS em qualquer realidade e por qualquer profissional. No entanto, apesar dos caminhos 
propostos para a assistência em Saúde Mental na APS, no contexto do Programa Nacional em Saúde Mental e da RAPS, intervenções terapêuticas não farmacológicas ainda são incipientes, comprovando que medidas de suporte no TMC precisam ser consolidadas.
Em relação as categorias que emergiram após a leitura dos estudos foram: Terapia Cognitiva Comportamental e Terapia de Resolução de Problemas (TRP), Socioterapia e Cuidados Colaborativos (CC).

Quadro 2: Caracterização dos estudos sobre intervenções terapêuticas exclusivamente não farmacológicas em TCM. São Paulo, SP. Brasil, 2018.

\begin{tabular}{|c|c|c|c|c|}
\hline $\begin{array}{c}\text { Ano / } \\
\text { Base de } \\
\text { dados }\end{array}$ & $\begin{array}{c}\text { Autor } \\
\text { (es) }\end{array}$ & Título & Objetivo e amostra & Conclusão \\
\hline $\begin{array}{l}\text { * } 2011 \\
\text { BMC } \\
\text { Public } \\
\text { Health }\end{array}$ & $\begin{array}{l}\text { ARAYA } \\
\text { R. et } \\
\text { al. }{ }^{12}\end{array}$ & $\begin{array}{l}\text { Problem-solving therapy } \\
\text { for depression and } \\
\text { common mental } \\
\text { disorders in Zimbabwe: } \\
\text { piloting a task-shifting } \\
\text { primary mental health } \\
\text { care intervention in a } \\
\text { population with a high } \\
\text { prevalence of people } \\
\text { living with HIV }\end{array}$ & $\begin{array}{l}\text { Adotar uma intervenção } \\
\text { de baixo custo para } \\
\text { TMC, adaptada } \\
\text { localmente, a partir de } \\
\text { Terapia de Resolução de } \\
\text { Problemas em um } \\
\text { ambiente africano. } \\
\text { Amostra: } 143 \text { pacientes }\end{array}$ & $\begin{array}{l}\text { Evidências de melhora } \\
\text { clínica significativa em } \\
\text { TMC quando associada à } \\
\text { Terapia de Resolução de } \\
\text { Problemas, podendo esta } \\
\text { ser adaptada localmente } \\
\text { e aplicada por } \\
\text { profissionais de saúde } \\
\text { leigos }\end{array}$ \\
\hline $\begin{array}{l}* * 2010 \\
\text { Journal } \\
\text { of } \\
\text { Affectiv } \\
\text { e } \\
\text { Disorder } \\
\text { s }\end{array}$ & $\begin{array}{l}\text { AFUW } \\
\text { APE, S. } \\
A, \text { et } \\
\text { al. }^{13}\end{array}$ & $\begin{array}{l}\text { The Cares of Life } \\
\text { Project (CoLP): An } \\
\text { exploratory randomized } \\
\text { controlled Trial of a } \\
\text { community-based } \\
\text { intervention for Black } \\
\text { people with common } \\
\text { mental disorder. }\end{array}$ & 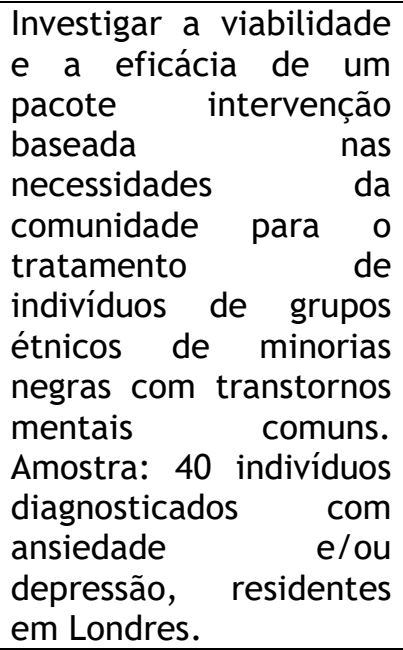 & $\begin{array}{l}\text { Intervenções psicossociais } \\
\text { efetivas e culturalmente } \\
\text { aceitas podem ser } \\
\text { aplicadas na comunidade, } \\
\text { para os indivíduos de } \\
\text { grupos étnicos, de } \\
\text { minoria negra com } \\
\text { ansiedade e depressão, } \\
\text { sem custo significativo. }\end{array}$ \\
\hline $\begin{array}{l}\text { *2011 } \\
\text { PLoS } \\
\text { ONE }\end{array}$ & $\begin{array}{l}\text { SCHOL } \\
\text { TE W. } \\
F, \quad \text { et } \\
\mathrm{al}^{14}\end{array}$ & $\begin{array}{l}\text { The effect on mental } \\
\text { health of a largescale } \\
\text { psychosocial } \\
\text { intervention } \\
\text { survivors of mass } \\
\text { violence: a quasi- } \\
\text { experimental study in } \\
\text { Rwanda }\end{array}$ & $\begin{array}{l}\text { Estudar o efeito sobre a } \\
\text { saúde mental da } \\
\text { intervenção psicossocial } \\
\text { Socioterapia, em larga } \\
\text { escala voltada, } \\
\text { principalmente, a } \\
\text { melhorar o vínculo social } \\
\text { na população em Ruanda } \\
\text { após o genocídio. } \\
\text { Amostra: } 73 \text { indivíduos } \\
\text { submetidos à avaliação de } \\
\text { TMC pelo Self Reporting } \\
\text { Questionnaire (SRQ-20) }\end{array}$ & $\begin{array}{l}\text { Os achados indicam que a } \\
\text { socioterapia pode ser } \\
\text { clinicamente relevante e } \\
\text { benéfica para casos de TMC. } \\
\text { A intervenção psicossocial } \\
\text { aplicável em grande escala, } \\
\text { voltada para o vínculo social, } \\
\text { trouxe melhora da saúde } \\
\text { mental em sobreviventes de } \\
\text { violência pós-conflito, e } \\
\text { pode ter efeito positivo em } \\
\text { outras populações com } \\
\text { demandas semelhantes. }\end{array}$ \\
\hline
\end{tabular}

Continua na página seguinte. 


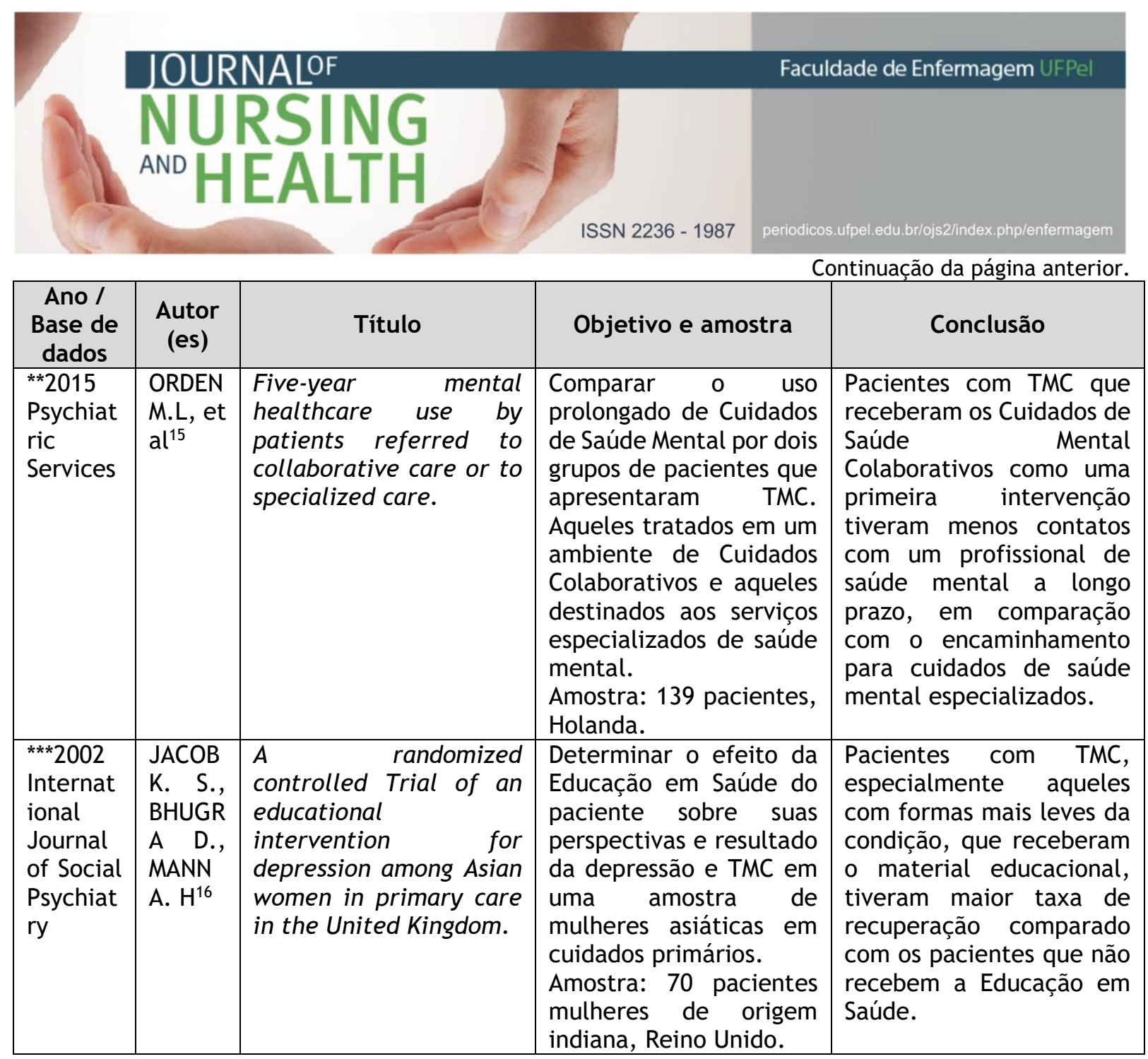

Fonte: Base de dados da Web of Science* ${ }^{*}$. Psycinfo ${ }^{* *}$ e CINHAL ${ }^{* * *}$

\section{DISCUSSÃO}

Os profissionais da APS se deparam a todo o momento com usuários em situação de sofrimento psíquico, e mesmo reconhecendo a importância dessa questão, as práticas em saúde mental suscitam muitas dúvidas, curiosidades e receios nos profissionais de saúde. ${ }^{7-8}$ Entretanto, é nesse cenário que pessoas com TMC procuram ser acolhidas, compreendidas e atendidas em suas necessidades.

Os casos de TMC muitas vezes são subdiagnosticados e subestimados pelos profissionais, devido à pouca capacitação técnica das equipes para melhor identificação e intervenção em pessoas em sofrimento psíquico. Isso representa um paradoxo, uma vez que a APS tem potência para desenvolver ações de promoção da saúde mental, conduzir rastreamento, encaminhamento e monitoramento destes pacientes. ${ }^{2}$

Assim, os profissionais que atuam nesse campo de conhecimento devem planejar e executar estratégias que possam atender a especificidade dessa clientela.

Nessa revisão, verificou-se que as intervenções não farmacológicas desenvolvidas no cenário da APS junto às pessoas com TMC são escassas, mostrando que há uma lacuna na produção científica que trate unicamente dessa perspectiva. Os estudos são, em sua maioria, conduzidos com amostras pequenas, 


\section{ISSN 2236 - 1987}

com população bastante específica, porém passível de reprodução em diferentes realidades, e não afirmam que as intervenções psicossociais testadas demonstram maiores resultados que as intervenções farmacológicas, contudo, trazem que são estratégias de baixo custo e de fácil implementação.

O Ministério da Saúde orienta que as intervenções em SM na APS devem gerar meios de modificação e qualificação das condições e modos de vida dos usuários, em respeito às suas singularidades, e que não se restrinjam à cura de doenças. ${ }^{7}$

Terapia

Cognitiva

Comportamental (TCC) e Terapia de Resolução de Problemas (TRP)

A TCC e a TRP são intervenções realizadas aos acometidos por TMC que se mostraram muito presentes nos estudos analisados. Tal popularidade se deve ao fato de serem intervenções mais baratas e que demonstram custobenefício satisfatório. ${ }^{17}$

A TCC é uma psicoterapia que auxilia os pacientes a superar seus problemas, identificando e desafiando pensamentos, comportamentos e reações emocionais. ${ }^{18-22}$ Dessa forma, a sua utilização tem como objetivo a percepção do paciente sobre 0 gerenciamento de pensamentos e comportamentos negativos.

Em contrapartida, a TRP focaliza a troca de experiência em grupo, tornando o diálogo e a interação social uma ferramenta para a descoberta de novas soluções para seus problemas a partir da partilha de vivências pessoais e da forma como os problemas ou até a doença afetam suas vidas. A discussão em grupo também favorece a construção de um pensamento mais crítico e reflexivo acerca da temática colocada em pauta. ${ }^{21-22}$

Os estudos ${ }^{18-22}$ revelam que a TCC é mais eficaz do que a TRP em relação à redução dos agravos de Saúde Mental, com destaque para a ansiedade, depressão e o estresse. Contudo, nota-se que a ansiedade é atenuada nas duas intervenções de forma bastante parecida, corroborando para o fato de que TCC e TRP são igualmente eficazes para o tratamento de ansiedade.

Em um trabalho realizado no Zimbabwe, ${ }^{12}$ o objetivo foi testar uma "Intervenção - O Banco da Amizade" de baixo custo para o sistema de saúde local, adaptada a partir de TRP e ministrada por mulheres leigas treinadas e supervisionadas para saber se era viável e possivelmente eficaz, bem como a melhor forma de intervenção à população acometida com TMC. A proposta foi desenvolvida em um subúrbio de alta densidade demográfica, caracterizado pela diversidade étnica, alto desemprego, comércio informal e taxa de alfabetização estimada em mais de $90 \%$.

Os atendimentos ocorreram nos jardins de clínicas de saúde mental da região, a maioria das sessões foi realizada com os participantes acomodados em um banco de madeira denominado "The Friendship Bench" (Os Bancos da Amizade) que foram feitos para o projeto por um artesão local. Foi realizada uma sessão semanal, de 30 a 45 minutos, durante seis semanas, incluindo uma ou duas 


\section{JOURNALOF \\ NURSING \\ ANo HEALTH}

ISSN 2236 - 1987

Project (CoLP). Um dos estudos desenvolvidos nesse projeto maior propôs investigar a viabilidade e eficácia de um pacote de intervenções psicossociais, levando em consideração a cultura dessa minoria e o custo que essas propostas de cuidados em saúde mental representariam, para que fossem reproduzíveis ou não em outras realidades. ${ }^{13}$

Baseado nos sistemas de crenças em relação ao TMC, o CoLP objetivou melhorar o estado de saúde percebido, o funcionamento psicossocial e a perspectiva diante dos eventos da vida diária. 0 pacote de intervenções psicossociais não farmacológicas aplicadas no estudo incluem: Aconselhamento ${ }^{23}$, Educação em Saúde ${ }^{24}$ e Mentoring, Advocacy, ${ }^{25}$ todos com base epistemológica na TCC e na Terapia Focada na Solução (TFS). ${ }^{13}$

\section{Enquanto}

abordagem psicoterapêutica reconhecida cientificamente, a TCC associa a teoria cognitiva com técnicas oriundas da análise do comportamento ${ }^{22} \mathrm{e}$ caracteriza-se por focar o presente, direcionando à solução de problemas atuais, mudanças de comportamentos e pensamentos disfuncionais. ${ }^{17,23}$ A TFS é ramificação da terapia familiar sistêmica, caracteriza-se por ser breve com média de duração de seis a dez sessões, com vistas a solucionar rapidamente as queixas e possibilitar maior satisfação com a vida. ${ }^{20,26}$

De acordo com a American Psychological Association (APA), o Aconselhamento é uma relação de ajuda, relativamente rápida, utilizada em processos disfuncionais individuais, familiares e de organizações, possibilitando a expressão e 


\section{NURSING \\ ANO HEALTH}

elaboração do indivíduo a respeito de suas experiências de modo compreensivo. ${ }^{23}$

O Aconselhamento é uma prática que considera os aspectos culturais do grupo em que o indivíduo está inserido e tem envergadura para ser utilizado com minorias étnicas e raciais, ajudando na tomada de decisão em situações objetivas, de acordo com os recursos e potencialidades pessoais que envolvam conflitos conscientes internos e externos em contexto de uma ansiedade considerada normal, ${ }^{23}$ a fim de promover bem-estar, respeito pelo outro e suas características sociais, culturais e históricas.

o "Advocacy" está associado à defesa dos direitos, apoio e proteção à autonomia, ${ }^{25}$ o qual busca tornar-se uma voz para os vulneráveis. 0 "Mentoring" caracteriza-se como um processo de aprendizagem no qual aquele mais experiente em determinada área de atuação, o mentor, repassa a alguém inexperiente, mentorado, conhecimentos, experiências, perspectivas e valores, adquiridos ao longo de sua vida, que serão proveitosos a outros menos experientes que buscam crescimento e desenvolvimento em tal área. ${ }^{20}$

Tem-se ainda, a Educação em Saúde que visa implementar hábitos saudáveis por meio da articulação entre o conhecimento técnico e popular, como também mobilizar alternativas individuais e coletivas. ${ }^{20} \mathrm{~A}$ promoção da educação em saúde propõe empoderar e auxiliar na transformação da realidade do indivíduo, para que esse possa
ISSN 2236 - 1987

deliberar sobre suas condições de saúde.

Todas as intervenções citadas acima são de suma importância, pois, contribuem para a redução do uso exclusivo de tratamentos farmacológicos, no que diz respeito ao TMC. E ainda, na conjuntura da APS é necessário olhar o indivíduo como um todo, não tratando apenas os sintomas, é necessário realizar um acompanhamento aprofundado sobre suas necessidades de saúde para que assim a assistência em saúde mental, e a escolha da melhor intervenção atinjam todos os âmbitos da vida do indivíduo, melhorando cada vez mais a assistência em saúde na APS.

As mazelas da má detecção e falha com o tratamento de desordens mentais comuns, foram objetos de estudo com mulheres asiáticas britânicas, a maioria revelou não aderir ao tradicional modelo médico/doença, devido ao estigma e medo da falta de confidencialidade do tratamento. Tal estudo abordou modelos explicativos, visão do paciente sobre a origem da doença, causas, consequências e expectativas de tratamento. Neste contexto as intervenções não farmacológicas ganham força, na garantia do verdadeiro sentido do cuidar. 8,24

\section{Socioterapia}

Estudo realizado em Rwanda ${ }^{14}$ buscou avaliar a intervenção psicossocial da socioterapia, em larga escala voltada, na saúde mental e melhorar o vínculo social de pessoas que vivenciaram o genocídio. 


\section{JOURNALOF \\ NURSING \\ AND HEALTH}

A socioterapia se fundamenta na interação entre o ambiente social e o indivíduo para estabelecer relações de caráter colaborativo, e tem como premissa que ao alcançar o respeito mútuo, confiança e cuidado na interação grupal, o indivíduo se fortalece mentalmente para enfrentar seus problemas, aumentando sua capacidade de resiliência e, consequentemente, a resolução de problemas. ${ }^{14,26}$

O programa de socioterapia é baseado na comunidade, isto é, é realizado por líderes comunitários treinados em Ruanda e familiarizados com a história local e é implementado em nível populacional. Como requisitos, os líderes deveriam exercer liderança compartilhada, comunicação bidirecional e consenso na tomada de decisões e ser receptivo para aprender com a experiência adquirida com a própria interação social. ${ }^{14}$

A intervenção foi útil para reduzir os agravos de TMC na população sobrevivente dos conflitos, contudo, as mulheres tiveram resultados superiores ao dos homens, possivelmente porque homens têm mais dificuldades em compartilhar seus problemas emocionais e, além disso, poderiam estar menos acometidos por TMC no início do tratamento. ${ }^{14}$

Após aplicação do Self-Reporting Questionnaire e participação em grupos de socioterapia, observou-se que os participantes relataram menos sintomas significativos de TMC comparado ao grupo controle, melhoria que persistiu após oito meses. 0 estudo destacou a importância da realização de grupos comunitários como terapêutica não-
ISSN $2236-1987$

farmacológica na APS, uma vez que as terapias comunitárias têm como objetivo propiciar alívio ao sofrimento emocional por meio do compartilhamento de experiências de vida, promovendo mudanças significativas nos pacientes e resultados positivos quando implementados com conhecimento técnico - científico e humanístico. ${ }^{14}$

No Brasil, a socioterapia tem sido desenvolvida em cenários de tratamento psiquiátrico para pessoas com transtorno mental, de caráter crônico, na perspectiva de criar espaços de sociabilidade e não foram encontrados estudos que abordassem a prática vinculada ao TMC na APS. 7,26

Ressalta-se a importância de estudos que destacam as intervenções frente aos TMC na realidade brasileira, dado à sua alta prevalência no país, principalmente, com o objetivo de amenizar o sofrimento psíquico, evitando a evolução para quadros mais graves ou ainda, a cronificação dos sintomas. ${ }^{24}$ Rwanda, nos últimos dez anos, trabalha com socioterapia ${ }^{26}$ e mostra como a comunidade pode apoiar-se mutuamente para lidar com o sofrimento individual e social, promover a saúde mental e o bemestar psicossocial.

\section{Cuidados Colaborativos}

0 cuidado colaborativo em instituições de saúde compreende: liderança, comunicação, confiança e integração. ${ }^{15}$ Para o desenvolvimento de um trabalho colaborativo e um ambiente harmônico, é impreterível que os profissionais de saúde estejam motivados a criar um meio favorável para a cooperação no planejamento, 
além da adequada coordenação das atividades em saúde mental. ${ }^{13-16}$

Trabalho desenvolvido na Holanda $^{15}$ com 165 pacientes de instituições locais de cuidados de Saúde Mental em Haia, comparou a eficácia de duas intervenções em saúde mental: Programa de Cuidados Colaborativos (PCC) e Cuidados Habituais $(\mathrm{CH})$ especializados em saúde mental.

Para a realização deste estudo, ${ }^{15}$ os pacientes foram distribuídos em dois grupos, 102 pacientes foram encaminhados para o PCC e 63 encaminhados para $\mathrm{CH}$. A intervenção prosseguiu da seguinte forma: os médicos destinados ao PCC forneciam informações acerca da saúde mental dos pacientes, os pacientes tinham livre acesso aos médicos especializados em Saúde Mental para que tivessem conhecimento de seus agravos de saúde e pudessem destinar o melhor tratamento. Após um ano de estudo, a hipótese de que a intervenção realizada com PCC é mais eficiente foi comprovada ao constatar que aqueles que fizeram uso desta intervenção tiveram menos contato com 0 profissional especializado em saúde mental quando comparados aqueles que foram encaminhados ao tratamento usual. ${ }^{15}$

Os pontos que favoreceram 0 sucesso do PCC se resumem na redução de sintomas psíquicos demonstrados pelos pacientes, como também no método intensivo de tratamento, capacitação e melhor habilidade na resolução de problemas. Contudo, para alcançar o proposto, é necessário que os pacientes sejam bastante ativos ao trabalhar seus problemas nas sessões de terapia. O fato de os intervalos entre as sessões aumentar ao transcorrer do tratamento auxilia o paciente a lidar de maneira mais independente em seu autocuidado, acarretando, consequentemente, em menor contato com o terapeuta, já que os eventos estressores da vida diária são encarados com maior resiliência. ${ }^{15}$

Estudo ${ }^{16}$ similar realizado na Ásia, com 70 pacientes classificados como casos de transtorno mental comum utilizando o General Health Questionnaire 12. Os 35 pacientes foram aleatoriamente distribuídos para receber educação sobre a natureza, causas, prevalência e tratamento da depressão e outors 35 não receberam tal informação. Não houve diferenças estatísticas entre os dois grupos nas características iniciais. Os indivíduos foram acompanhados por dois meses. No entanto, pacientes com transtornos mentais comuns, especialmente aqueles com formas mais leves da doença, que receberam o material educacional tiveram uma taxa de recuperação mais alta do que os pacientes que não receberam tal educação. ${ }^{16}$

Desta forma, é importante ressaltar que a comunicação/educação é um elemento fundamental para o sucesso do trabalho colaborativo. É através da comunicação clara e eficiente que se mantém boa relação entre paciente e equipe multiprofissional.

Outro estudo, traz informações coletadas em um grupo focal realizado entre os asiáticos britânicos com TMC ${ }^{19}$ serviu de base para a elaboração do conteúdo do material que continha a descrição dos sintomas típicos de 


\section{ISSN $2236-1987$}

depressão e ansiedade, com algumas causas comuns, e demonstrava que um terço da população é acometida com um desses sintomas em algum ponto da vida. Contudo, o folheto ressaltou que os sintomas podem ser causados por vários fatores de estresse, mas são sua prevalência e gravidade associadas à desordem psicológica. Por fim, houve o aconselhamento do paciente para que este entrasse em contato com os médicos para que as opções de tratamento fossem discutidas. ${ }^{19}$

$\begin{array}{cc}\text { Neste } & \text { contexto, tem-se a } \\ \text { integração } & \text { através }\end{array}$
aconselhamento, que é compreendido como os esforços empreendidos para manter as atividades e relações entre os diversos membros de forma coesa, respeitando a autonomia de cada um e reconhecendo sua interdependência, traz benefícios para o paciente e para os profissionais de saúde desenvolvendo habilidades, conhecimentos e respeito às diferenças. ${ }^{19}$

\section{CONSIDERAÇÕES FINAIS}

Com a realização da presente pesquisa foi possível evidenciar uma lacuna de publicações que oriente a importância de se constituir mais práticas de saúde mental voltada às intervenções não farmacológicas no Transtorno Mental Comum. Tal fato dáse, como demonstrado anteriormente, pela alta prevalência da população que é acometida por TMC, apresentando situações de extremo sofrimento psíquico e com dificuldade de diagnóstico por profissionais não especializados em Saúde Mental.

As categorias de intervenções não farmacológicas encontradas nesta revisão integrativa que se mostraram efetivas a pessoas acometidas por TMC foram: a terapia cognitiva comportamental, terapia de resolução de problemas, socioterapia e cuidados colaborativos. Tais intervenções visam amenizar sofrimentos físicos e mentais, evitando-se, assim, a cronificação dos sintomas. Estes resultados podem direcionar a seleção de intervenções a serem implementadas pelos profissionais de saúde generalistas e especialistas em Saúde Mental, com foco na qualidade e resolutividade da assistência prestada.

\section{REFERÊNCIAS}

1 Organização Mundial da Saúde (OMS). Integração da saúde mental nos cuidados de saúde primários: uma perspectiva global [Internet]. Lisboa; 2008[acesso em 2019 mar 04]. Disponível em: http://www.who.int/eportuguese/pu blications/Integracao_saude_mental_c uidados_primarios.pdf

2 Saraceno B, Van Ommeren M, Batniji R, Cohen A, Gureje O, Mahoney J, et al. Barriers to improvement of mental health services in low-income and middle in some countries. J. Lancet. [Internet]. 2007[cited 2019 Mai 01];370(9593):1164-74. Available from:

https: / /www.thelancet.com/journals /lancet/article/PIIS0140-

6736(07)61263-X/fulltext

3 Goldberg D, Huxley P. Common mental disorders: a bio-social model. London: Routledge; 1992.

4 Lucchese R, Sousa K, Bonfin SP, Vera I, Santana FR. Prevalence of common mental disorders in primary health 


\section{JOURNALOF

care. Acta Paul. Enferm. (Online). [Internet]. 2014[cited 2019 Mar 04];2017(3):200-7. Available from: http://www.scielo.br/pdf/ape/v27n3 /en_1982-0194-ape-027-003-0200.pdf

5 Rocha SV, Almeida MMG, Araújo TM, Virtuoso Júnior JS. Prevalence of common mental disorders among the residents of urban areas in Feira de Santana, Bahia. Rev. bras. epidemiol. [Internet]. 2010[cited 2019 Mar 04];13(4):630-40. Available from: http://www.scielo.br/pdf/rbepid/v13 n4/en_08.pdf

6 Skapinakis $P$, Bellos S, Koupidis S, Grammatikopoulos L, Theodorakis PN, Mavreas V. Prevalence and sociodemographic associations of common mental disorders in a national lyre present ative sample of the general population of Greece. BMC Psychiatry [Internet]. 2013[cited 2019 Mar 04];13(163):1-14. Available from: https://bmcpsychiatry.biomedcentral .com/articles/10.1186/1471-244X-13163

7 Vidal CEL, Yañez BFP, Chaves CVS, Yañez CFP, Michalaros IA, Almeida LAS. Transtornos mentais comuns e uso de psicofármacos em mulheres. Cad. saúde colet., (Rio J.). [Internet]. 2013[acesso em 2019 mar 04];21(4):457-64. Disponível em: http://www.scielo.br/pdf/cadsc/v21n 4/v21n4a15.pdf

8 Fonseca MLG, Guimarães MBL, Vasconcelos EM. Sofrimento difuso e transtornos mentais comuns: uma revisão bibliográfica. Rev. APS. [Internet]. 2008[acesso em 2019 mar 04];11(3):285-94. Disponível em: http://ojs2.ufjf.emnuvens.com.br/ap s/article/view/14269/7719
9. Soares $C B$, Hoga LAK, Peduzzi $M$, Sangaleti C, Yonekura T, Silva DRAD. Integrative review: concepts and methods used in nursing. Rev. Esc. Enferm. USP. [Internet]. 2014[cited 2019 Mar 04];48(2):329-39. Disponível em:

http://www.scielo.br/pdf/reeusp/v48 n2/0080-6234-reeusp-48-02-335.pdf

10 Souza MT, Silva MD, Carvalho R. Revisão integrativa: o que é e como fazer. Einstein (São Paulo). [Internet]. 2010[acesso em 2019 mar 04];8(1):1026. Disponível em: http://www.scielo.br/pdf/eins/v8n1/ pt_1679-4508-eins-8-1-0102.pdf

11 Santos CMC, Pimenta CAM, Nobre MRC. The PICO strategy for there search question construction and evidence search. Rev. latinoam. enferm. (Online). [Internet]. 2007[cited 2019 Mar 04];15(3):508-11. Available from: http://www.scielo.br/pdf/rlae/v15n3 /v15n3a23.pdf

12 Chibanda D, Mesu P, Kajawu L, Cowan F, Araya R, Abas MA. Problemsolving therapy for depression and common mental disorders in Zimbabwe: piloting a task-shifting primary mental health care intervention in a population with a high prevalence of people living with HIV. BMC Public Health [Internet]. 2011[cited 2019 Mar 04];11(828):14712445. Available from: https: / /bmcpublichealth. biomedcentr al.com/articles/10.1186/1471-245811-828

13 Afuwape SA, Craig TK, Harris T, Clarke M, Flood A, Olajide D, et al. The caresof life project (CoLP): an exploratory randomised controlled 


\section{t}

trial of a community-based intervention for black people with common mental disorder. J Affect Disord [Internet]. 2010[cited 2018 Nov 30];127(1-3):370-4. Available from: https://www.ncbi.nlm.nih.gov/pubm ed/20547421

14 Scholte WF, Verduin F, Kamperman AM, Rutayisire T, Zwinderman $\mathrm{AH}$, Stronks K. The effect on mental health of a larg escale psycho social intervention for survivors of mass violence: A quasi-experimental study in Rwanda. PLoS One [Internet]. 2011[cited 2019 Mar 04];6(8):218-9. Available from: https: / / journals.plos.org/plosone/arti cle?id=10.1371/journal. pone.0021819

15 Orden ML, Deen ML, Spinhoven P, Haffmans J, Hoencamp E. Five-year mental healthcare use by patients referred to collaborative care or to specialized care. Psychiatr Serv [Internet]. 2015[cited 2019 Mar 04]. Available from: https://ps.psychiatryonline.org/doi/1 0.1176/appi.ps.201400238

16 Jacob KS, Bhugra D, Mann AH. A randomised controlled trial of an educational intervention for depression among Asian women in primary care in the United Kingdom. Int J Soc Psychiatry [Internet]. 2002[cited 2019 Apr 19];48(2):139-48. Available from: https://journals.sagepub.com/doi/ab s/10.1177/002076402128783190

17 Blonk RWB, Brenninkmeijer V, Lagerveld SE, Houtman ILD. Return to work: a comparison of two cognitive behavioural interventions in cases of work-related psychological complaints among the self-employed. Work Stress
[Internet]. 2006[cited 2019 Apr 19];20(2):129-44. Available from: https://www.researchgate.net/public ation/253919110_Return_to_work_A_c omparison_of_two_cognitive_behaviou ral_interventions_in_cases_of_workrelated_psychological_complaints_am ong_the_self-employed

18 Hofmann SG, Asnaani A, Vonk IJJ, Sawyer AT, Fang A. The efficacy of cognitive behavioral therapy: a review of meta-analyses. Cognit Ther Res [Internet]. 2012[cited 2019 Mar 04];36(5):427-40. Available from: https://www.ncbi.nlm.nih.gov/pmc/a rticles/PMC3584580/pdf/nihms394950 .pdf

19 Singulane BAR, Sartes LM. Aliança terapêutica nas terapias cognitivocomportamentais por vídeo conferência: uma revisão da literatura. Psicol. ciênc. prof. [Internet]. 2017[acesso em 2019 mar 04];37(3):784-98. Disponível em: http://www.scielo.br/pdf/pcp/v37n3 /1982-3703-pcp-37-3-0784.pdf

20 Lemes CB, Ondere Neto J. Aplicações da psicoeducação no contexto da saúde. Temas psicol. (Online). [Internet]. 2017[acesso em 2019 mar 04];25(1):17-28. Disponível em:

http://pepsic.bvsalud.org/pdf/tp/v25 n1/v25n1a02.pdf

21 Araya R, Flynn T, Rojas G, Fritsch R, Simon G. Cost-effectiveness of a primary care treatment program for depression in low-income women in Santiago, Chile. Am J Psychiatry [Internet]. 2006[cited 2019 Mar 04];163(8):1379-87. Available from: https://ajp.psychiatryonline.org/doi/ full/10.1176/ajp.2006.163.8.1379?url_ 


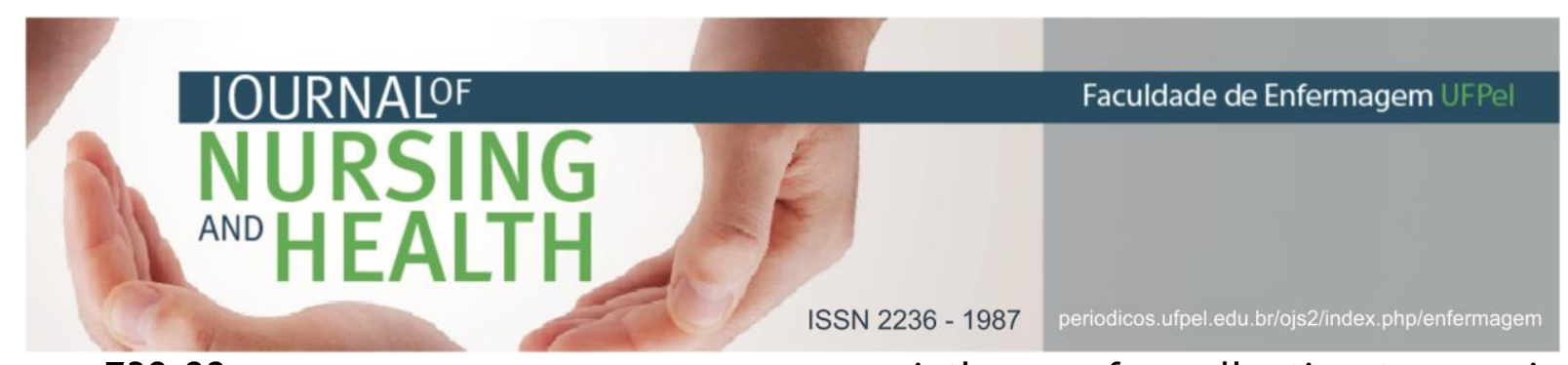

ver $=$ Z39.88-

2003\&rfr_id=ori:rid:crossref.org\&rfr_d at=cr_pub\%3dpubmed

22 Brandtner $M$, Serralta FB. Terapia Cognitivo-Comportamental para compras compulsivas: um estudo de caso sistemático. Psicol. teor. pesqui. [Internet]. 2016 [acesso em 2019 mar 04]; 32(1):181-8. Disponível em: http://www.scielo.br/pdf/ptp/v32n1 / 1806-3446-ptp-32-01-00181.pdf

23 Scorsolini-Comin F. Aconselhamento psicológico: práticas e pesquisas nos contextos nacional e internacional. Rev. Subj. (Online). [Internet]. 2015[acesso em 2019 mar 04];15(1):130-41. Disponível em: http://pepsic. bvsalud.org/pdf/rs/v15 n1/15.pdf

24 Sehgal R, Saules K, Young A, Grey MJ, Gillem AR, Nabors NA. Practicing what we know: multicultural counseling competence among clinical Psychology trainees and experienced multicultural psychologists. Cultur Divers Ethnic Minor Psychol [Internet]. 2011[cited 2019 Apr 19];17(1):1-10. Available from: https: / / psycnet.apa.org/record/201103115-001

25 Kalaitzidis E, Jewell $P$. The concept of advocacy in nursing: a critical analysis. Health Care Manag (Frederick) [Internet]. 2015[cited 2019 Apr 19];34(4):308-15. Available from: https://journals.lww.com/healthcare managerjournal/Abstract/2015/10000 /The_Concept_of_Advocacy_in_Nursin g_A_Critical.6.aspx

26 Jansen S, White R, Hogwood J, Jansen A, Gishoma D, Mukamana D, et al. The "treatment gap" in global mental health reconsidered: sociotherapy for collective trauma in Rwanda. Eur J Psychotraumatol [Internet]. 2015[cited 2019 Mar 04];6:1-6. Available from: https://www.ncbi.nlm.nih.gov/pmc/a rticles/PMC4654767/pdf/EJPT-6-

28706.pd

Data de submissão: 11/10/2018

Data de aceite: 15/04/2019

Data de publicação: 14/05/2019 